\title{
THE HIGHER ORDER COMMUTATORS OF THE FRACTIONAL INTEGRALS ON HARDY SPACES
}

\author{
SHUNCHAO LONG ${ }^{\Xi}$ and JIAN WANG
}

(Received 15 November 2000; revised 24 March 2004)

Communicated by A. H. Dooley

\begin{abstract}
In this paper we investigate the boundedness on Hardy spaces for the higher order commutator $T_{b, m}^{\tau}$ generated by the $B M O$ function $b$ and fractional integral type operator $T^{\mathfrak{r}}$, and establish the boundedness theorems for $T_{b, m}^{\tau}$ from $H_{b, m}^{p_{1}, q_{1}, s}$ to $L^{p_{2}}$ and to $H^{p_{2}}\left(0<p_{1} \leq 1\right)$, and from $H \dot{K}_{q_{1}, b, m}^{\alpha, p_{1}, s}$ to $\dot{K}_{q_{2}}^{\alpha, p_{2}}$ and to $H \dot{K}_{q_{2}}^{\alpha, p_{2}}$, respectively, for certain ranges of $\alpha, p_{1}, q_{1}, p_{2}, q_{2}$ and $s$.

2000 Mathematics subject classification: primary 42B20; secondary 42B30.

Keywords and phrases: Hardy space, Herz space, commutator, fractional integral type operator, $B M O$.
\end{abstract}

\section{Introduction}

Let $T$ be a linear operator and $b$ a $B M O$ function. The higher order commutator operators are defined as

$$
T_{b, m} f(x)=T\left((b(\cdot)-b(x))^{m} f(\cdot)\right)(x), \quad m=0,1,2, \ldots
$$

Obviously, $T_{b, 0}=T, T_{b, 1}=[b, T]$ which is the commutator in [6], and

$$
T_{b, m}=\left[b, T_{b, m-1}\right], \quad m=1,2, \ldots
$$

Coifman, Rochberg and Weiss [6] stated that if $T$ is a Calderón-Zygmund singular integral operator, then $T_{b, 1}=[b, T]$ is bounded on $L^{p}\left(\mathbb{R}^{n}\right)$ for $1<p<\infty$. Chanillo [4] extended this result to the fractional integral. Subsequently, many authors have

The authors were supported by the Foundation of Province Educational Committee of Hunan (99B03) and the Youth Foundation of National Natural Science Foundation of China (10101022) and Natural Science Foundation of Hunan Province (04JJ3037).

(C) 2005 Australian Mathematical Society $1446-7887 / 05 \$ A 2.00+0.00$ 
studied the $L^{p}$-boundedness of the commutator $T_{b, 1}=[b, T]$ (see, for example, [2, $3,10,22])$ and the higher order commutators $T_{b, m}, m=0,1, \ldots,($ see $[8,12,15,23])$. The case $0<p \leq 1$ was also considered by many authors. When $T$ is a CalderónZygmund singular integral operator, Perez [21], Pluszynski [20] and Alvarez [1] showed that $[b, T]$ does not map $H^{p}\left(\mathbb{R}^{n}\right)$ into $L^{p}\left(\mathbb{R}^{n}\right)$. However, Perez [21] proved that $T_{b, m}$ maps the modified spaces $H_{b, m}^{1, \infty, 0}\left(\mathbb{R}^{n}\right)$ to $L^{1}\left(\mathbb{R}^{n}\right)$, Alvarez [1] obtained that $[b, T]$ maps $H_{b, 1}^{p, \infty, 0}\left(\mathbb{R}^{n}\right)$ to $L^{p}\left(\mathbb{R}^{n}\right)$, and Long and Wang [15] proved that $T_{b, m}$ maps $H_{b, m}^{p, q, s}\left(\mathbb{R}^{n}\right)$ to $L^{p}\left(\mathbb{R}^{n}\right)$ and to $H^{p}\left(\mathbb{R}^{n}\right)$. In this paper, we will extend these results to the higher order commutators $T_{b, m}^{\tau}$ of fractional integral type operators $T^{\tau}$ and consider their boundedness from $H_{b, m}^{p_{1}, q_{1}, s}\left(\mathbb{R}^{n}\right)$ to $L^{p_{2}}\left(\mathbb{R}^{n}\right)$ and to $H^{p_{2}}\left(\mathbb{R}^{n}\right)$ for $0<p_{1} \leq 1$.

On the other hand, Herz type Hardy spaces were recently studied by many authors (see $[5,9,11,12,16-19])$. The boundedness of some operators on Herz spaces and Herz type Hardy spaces can be found in $[11,12,14-17,19]$. If $T$ is a standard Calderón-Zygmund operator, $[b, T]$ is bounded on Herz space $\dot{K}_{q}^{\alpha, p}\left(\mathbb{R}^{n}\right)$ (or $K_{q}^{\alpha, p}\left(\mathbb{R}^{n}\right)$ ) for $-n / q \leq \alpha<n(1-1 / q)$, but not for $\alpha \geq n(1-1 / q)$ (see [11,19]). It is not bounded even from $H \dot{K}_{q}^{\alpha, p, 0}\left(\mathbb{R}^{n}\right)$ (or $H K_{q}^{\alpha, p, 0}\left(\mathbb{R}^{n}\right)$ ) into $\dot{K}_{q}^{\alpha, p}\left(\mathbb{R}^{n}\right)$ (or $K_{q}^{\alpha, p}\left(\mathbb{R}^{n}\right)$ ) for $\alpha \geq n(1-1 / q)$. However, $[b, T]$ is bounded from $H \dot{K}_{q, b}^{\alpha, p, 0}\left(\mathbb{R}^{n}\right)$ (or $H K_{q, b}^{\alpha, p, 0}\left(\mathbb{R}^{n}\right)$ ) into $\dot{K}_{q}^{\alpha, p}\left(\mathbb{R}^{n}\right)$ (or $K_{q}^{\alpha, p}\left(\mathbb{R}^{n}\right)$ ) for $n(1-1 / q) \leq \alpha<n(1-1 / q)+\gamma$ (see [19]), just as the cases involving the standard Hardy space $H^{1}\left(\mathbb{R}^{n}\right)$ and the Lebesgue space $L^{1}\left(\mathbb{R}^{n}\right)$. Long and Wang [15] obtained the boundedness of the higher order commutators $T_{b, m}$ of the Calderón-Zygmund singular integral operators $T$ from $H \dot{K}_{q, b, m}^{\alpha, p, s}\left(\mathbb{R}^{n}\right)$ (or $H K_{q, b, m}^{\alpha, p, s}\left(\mathbb{R}^{n}\right)$ ) into $\dot{K}_{q}^{\alpha, p}\left(\mathbb{R}^{n}\right)\left(\right.$ or $\left.K_{q}^{\alpha, p}\left(\mathbb{R}^{n}\right)\right)$, and from $H \dot{K}_{q, b, m}^{\alpha, p, s}\left(\mathbb{R}^{n}\right)$ (or $H K_{q, b, m}^{\alpha, p, s}\left(\mathbb{R}^{n}\right)$ ) into $H \dot{K}_{q}^{\alpha, p}\left(\mathbb{R}^{n}\right)$ (or $H K_{q}^{\alpha, p}\left(\mathbb{R}^{n}\right)$ ) for some ranges of $p, q, s$ and $\alpha$. The boundedness of higher order commutators of fractional integrals on Herz spaces was obtained for a range of $\alpha$ in [12]. Here, we will also investigate the boundedness for the higher order commutators $T_{b, m}^{\boldsymbol{r}}$ of the fractional integral type operators $T^{\boldsymbol{\tau}}$ from the Herz type Hardy spaces $H \dot{K}_{q_{1}, b, m}^{\alpha, p_{1}, s}\left(\mathbb{R}^{n}\right)$ (or $H K_{q_{1}, b, m}^{\alpha, p_{1}, s}\left(\mathbb{R}^{n}\right)$ ) to Herz spaces $\dot{K}_{q_{2}}^{\alpha, p_{2}}\left(\mathbb{R}^{n}\right)$ (or $K_{q_{2}}^{\alpha, p_{2}}\left(\mathbb{R}^{n}\right)$ ) and from $H \dot{K}_{q_{1}, b, m}^{\alpha, p, s}\left(\mathbb{R}^{n}\right)$ (or $H K_{q_{1}, b, m}^{\alpha, p_{1}, s}\left(\mathbb{R}^{n}\right)$ ) to $H \dot{K}_{q_{2}}^{\alpha, p_{2}}\left(\mathbb{R}^{n}\right)$ (or $H K_{q_{2}}^{\alpha, p_{2}}\left(\mathbb{R}^{n}\right)$ ) for certain ranges of $\alpha, p_{1}, q_{1}, p_{2}, q_{2}$ and $s$.

Let us introduce some definitions below.

Definition 1. Let $0 \leq \tau<n, 0<\gamma \leq 1, s \in \mathbb{N} \cup\{0\}, 1<q_{1} \leq q_{2}<\infty$ be such that $1 / q_{1}-1 / q_{2}=\tau / n$. $T^{\tau}$ is said to be a $\left(q_{1}, \tau ; s, \gamma\right)$-fractional integral type operator if $T^{\tau}$ is a bounded singular integral operator from $L^{q_{1}}\left(\mathbb{R}^{n}\right)$ into $L^{q_{2}}\left(\mathbb{R}^{n}\right)$ with kernel $K(x, y)$, which is $C^{\infty}$ away from the origin and satisfies the following conditions:

(i) $T^{r} f(x)=\int_{\mathbb{R}^{n}} K(x, y) f(y) d y$, if $x \neq y$;

(ii) $\left|\frac{\partial^{\zeta} K(x, y)}{\partial y^{\zeta}}-\frac{\partial^{\zeta} K\left(x, y^{\prime}\right)}{\partial y^{\zeta}}\right| \leq C_{\zeta} \frac{\left|y-y^{\prime}\right|^{\gamma}}{|x-y|^{n-\tau+s+\gamma}}$, if $|x-y| \geq 2\left|y-y^{\prime}\right|$, where $\zeta=\left(\zeta_{1}, \ldots, \zeta_{n}\right)$ is any multi-index and $s=|\zeta|=\zeta_{1}+\cdots+\zeta_{n}$. 
Denote by $[r]$ the integer part of the real number $r$. For $\beta=\left(\beta_{1}, \ldots, \beta_{n}\right) \in$ $(\mathbb{N} \cup\{0\})^{n}, x^{\beta}=x_{1}^{\beta_{1}} \cdots x_{n}^{\beta_{n}}$ and $|\beta|=\beta_{1}+\cdots+\beta_{n}$. Let

$$
\|f\|_{L^{q}\left(\mathbb{R}^{n}\right)}=\left(\int_{\mathbf{R}^{n}}|f(x)|^{q} d x\right)^{1 / q} .
$$

Denote by $T^{*}$ the conjugate operator of $T$.

DEFINITION 2. Let $0<p \leq 1 \leq q \leq \infty, p<q$, $[n(1 / p-1)] \leq s<\infty$. A function $a(x)$ is said to be a $(p, q, s ; b)$-atom of order $m$ if there exists a ball $B$ for which

(i) $\operatorname{supp} a \subseteq B=B\left(x_{0}, r\right)=\left\{x:\left|x-x_{0}\right|<r\right\}$;

(ii) $\|a\|_{L^{q}\left(\mathbb{R}^{n}\right)} \leq|B|^{1 / q-1 / p}$;

(iii) $\int_{\mathbb{R}^{n}} a(x) b^{i}(x) x^{\beta} d x=0 ;|\beta| \leq s, i=0,1, \ldots, m$.

Definition 3. Let $0<p \leq 1 \leq q \leq \infty, p<q,[n(1 / p-1)] \leq s<\infty$. We define $f \in H_{b, m}^{p, q, s}\left(\mathbb{R}^{n}\right)$ if and only if $f(x)=\sum_{k \in \mathbb{N}} \lambda_{k} a_{k}(x)$, where each $a_{k}$ is a $(p, q, s ; b)$-atom of order $m, \sum_{k \in \mathbb{N}}\left|\lambda_{k}\right|^{p}<+\infty$, and $\|f\|_{H_{b, m}^{p . q . s}\left(\mathbb{R}^{n}\right)} \sim\left(\sum_{k \in \mathbb{N}}\left|\lambda_{k}\right|^{p}\right)^{1 / p}$.

Obviously, $H_{b, m}^{1, \infty, 0}\left(\mathbb{R}^{n}\right)$ are the spaces $H_{b, m}^{1}\left(\mathbb{R}^{n}\right)$ which were introduced by Perez in [21]. By the atomic decomposition theory of Coifman and Weiss [7,13,25], if $0<p \leq 1 \leq q \leq \infty, p<q$ and $[n(1 / p-1)] \leq s<\infty$, it is easy to see that $H_{b, 0}^{p, q, s}\left(\mathbb{R}^{n}\right)=H^{p}\left(\mathbb{R}^{n}\right)$, the classical Hardy spaces.

THEOREM 1.1. Let $0<p_{1} \leq 1,1 / p_{2}=1 / p_{1}-\tau / n, 0<\gamma \leq 1, s>\left[n\left(1 / p_{1}-1\right)\right]$, $1<q_{1} \leq \infty$, and let $T^{\tau}$ be a $\left(q_{1}, \tau ; s, \gamma\right)$-fractional integral type operator (as in Definition 1) and $b \in B M O$. If $n /(n-\tau+s+\gamma)<p_{2}<+\infty$ and $0 \leq \tau<n$, then $T_{b, m}^{\tau}$ maps $H_{b, m}^{p_{1}, q_{1}, s}\left(\mathbb{R}^{n}\right)$ into $L^{p_{2}}\left(\mathbb{R}^{n}\right)$.

REMARK 1. Theorem 1.1 is equivalent to [21, Theorem 1.9] when $\tau=0, p_{1}=$ $p_{2}=1, s=0, q_{1}=\infty$; and to [1, Theorem 1.5] when $\tau=0, m=1, s=0$.

THEOREM 1.2. Let $0<p_{1} \leq 1,1 / p_{2}=1 / p_{1}-\tau / n, 0<\gamma \leq 1, s>\left[n\left(1 / p_{1}-1\right)\right]$, $1<q_{1} \leq \infty$, and let $T^{\tau}$ be a $\left(q_{1}, \tau ; s, \gamma\right)$-fractional integral type operator and $b \in$ BMO. Assume that $\left(T^{\tau}\right)^{*}\left(g_{i, \beta}\right)=C$ (a constant), $g_{i, \beta}(x)=b^{i}(x) x^{\beta},|\beta| \leq s, i=$ $0,1, \ldots, m$. If $n /(n-\tau+s+\gamma)<p_{2} \leq 1$ and $0 \leq \tau<\gamma$, then $T_{b, m}^{\tau}$ maps $H_{b, m}^{p_{1}, q_{1}, s}\left(\mathbb{R}^{n}\right)$ into $H^{p_{2}}\left(\mathbb{R}^{n}\right)$.

Let $B_{k}=\left\{x \in \mathbb{R}^{n}:|x| \leq 2^{k}\right\}, C_{k}=B_{k} \backslash B_{k-1}$, and $\chi_{k}=\chi_{C_{k}}$ for $k \in \mathbb{Z}$, where $\chi_{C_{k}}$ is the characteristic function of set $C_{k}$.

DefinITION 4. Let $0<\alpha<\infty, 0<p<\infty, 1 \leq q<\infty$. The Herz spaces are defined by 
(a) $\dot{K}_{q}^{\alpha, p}\left(\mathbb{R}^{n}\right)=\left\{f \in L_{\text {loc }}^{q}\left(\mathbb{R}^{n} \backslash\{0\}\right):\|f\|_{\dot{K}_{q}^{\alpha . p}\left(\mathbb{R}^{n}\right)}<+\infty\right\}$ (homogeneous space), where

$$
\|f\|_{\dot{K}_{q}^{\alpha, p}\left(\mathbf{R}^{n}\right)}=\left(\sum_{k \in \mathbf{Z}}\left|B_{k}\right|^{\alpha p / n}\left\|f \chi_{k}\right\|_{L^{q}\left(\mathbb{R}^{n}\right)}^{p}\right)^{1 / p},
$$

(b) $K_{q}^{\alpha, p}\left(\mathbb{R}^{n}\right)=L^{q}\left(\mathbb{R}^{n}\right) \cap \dot{K}_{q}^{\alpha, p}\left(\mathbb{R}^{n}\right)$ (non-homogeneous space), and $\|f\|_{K_{q}^{\alpha, p}\left(\mathbb{R}^{n}\right)}=$ $\|f\|_{L^{q}\left(\mathbf{R}^{n}\right)}+\|f\|_{\dot{K}_{q}^{\alpha, p}\left(\mathbb{R}^{n}\right)}$.

Obviously, $\dot{K}_{p}^{0, p}\left(\mathbb{R}^{n}\right)=L^{p}\left(\mathbb{R}^{n}\right)=K_{p}^{0, p}\left(\mathbb{R}^{n}\right)$ for all $0<p \leq \infty$.

Definition 5. Let $-\infty<\alpha<\infty, 0<p<\infty, 1 \leq q<\infty$ and $s \in \mathbb{N} \cup\{0\}$. A function $a(x)$ is said to be an $m$ th order central $H \dot{K}-(\alpha, q, ; b)_{s}$-atom if $a(x)$ satisfies

(i) $\operatorname{supp} a \subseteq B(0, r)=\left\{x \in \mathbb{R}^{n}:|x|<r, r>0\right\}$;

(ii) $\|a\|_{L^{9}\left(\mathbb{R}^{n}\right)} \leq|B(0, r)|^{-\alpha / n}$;

(iii) $\int_{\mathbf{R}^{n}} a(x) b^{i}(x) x^{\beta} d x=0,|\beta| \leq s, i=0,1, \ldots, m$.

A function $a(x)$ is said to be an $m$ th order central $H K-(\alpha, q, ; b)_{s}$-atom if $a(x)$ satisfies (ii), (iii) and

(i') $\operatorname{supp} a \subseteq \overline{B(0, r)}=\left\{x \in \mathbb{R}^{n}:|x|<r, r>1\right\}$.

DEFINITION 6. Let $0<p<\infty, 1<q<\infty, n(1-1 / q) \leq \alpha<\infty$ and $s \in \mathbb{N} \cup\{0\}$. We define $f \in H \dot{K}_{q, b, m}^{\alpha, p, s}\left(\mathbb{R}^{n}\right)$ (or $H K_{q, b, m}^{\alpha, p, s}\left(\mathbb{R}^{n}\right)$ ) if and only if $f(x)=\sum_{k \in \mathbb{Z}} \lambda_{k} a_{k}(x)$ (or $f(x)=\sum_{k \geq 0} \lambda_{k} a_{k}(x)$ ), where each $a_{k}$ is an $m$ order central $H \dot{K}-$ (or $H K-$ ) $(\alpha, q, ; b)_{s}$-atom with the support $B_{k}, \sum_{k \in \mathbb{Z}}\left|\lambda_{k}\right|^{p}<+\infty$ (or $\left.\sum_{k \geq 0}\left|\lambda_{k}\right|^{p}<+\infty\right)$, and

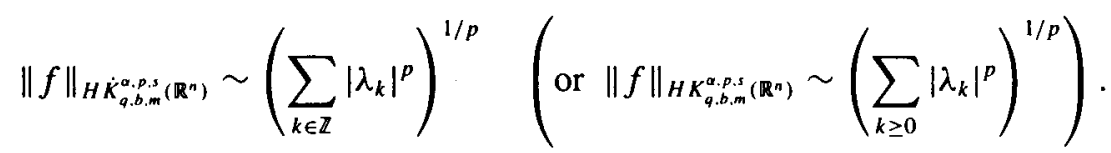

If $0<p<\infty, 1<q<\infty, n(1-1 / q) \leq \alpha<\infty$ and $s \geq[\alpha-n(1-1 / q)]$, it is easy to see that $H \dot{K}_{q, b, 0}^{\alpha, p, s}\left(\mathbb{R}^{n}\right)=H \dot{K}_{q}^{\alpha, p}\left(\mathbb{R}^{n}\right), H K_{q, b, 0}^{\alpha, p, s}\left(\mathbb{R}^{n}\right)=H K_{q}^{\alpha, p}\left(\mathbb{R}^{n}\right)$ (see $[11,17]$ or $[18,19])$. For $0<p<\infty, H \dot{K}_{p}^{0, p}\left(\mathbb{R}^{n}\right)=H K_{p}^{0, p}\left(\mathbb{R}^{n}\right)$ which are the usual Hardy spaces $H^{p}\left(\mathbb{R}^{n}\right)$. In particular, $H^{p}\left(\mathbb{R}^{n}\right)=L^{p}\left(\mathbb{R}^{n}\right)$ when $p>1$. When $1<q<\infty,-n / q<\alpha<n(1-1 / q)$ and $0<p \leq \infty, H \dot{K}_{q}^{\alpha, p}\left(\mathbb{R}^{n}\right)=\dot{K}_{q}^{\alpha, p}\left(\mathbb{R}^{n}\right)$ and $H K_{q}^{\alpha, p}\left(\mathbb{R}^{n}\right)=K_{q}^{\alpha, p}\left(\mathbb{R}^{n}\right)$ (see $\left.[11]\right)$.

THEOREM 1.3. Let $0 \leq \tau<n, s \in \mathbb{N} \cup\{0\}, 0<\gamma \leq 1,0<p_{1} \leq p_{2}<\infty$, $1<q_{1}<\infty, 1 / q_{2}=1 / q_{1}-\tau / n, n\left(1-1 / q_{1}\right) \leq \alpha<s+\gamma+n\left(1-1 / q_{1}\right)$, and let $b \in B M O$ and $T^{\tau}$ be $a\left(q_{1}, \tau ; s, \gamma\right)$-fractional integral type operator. Then $T_{b, m}^{\tau}$ maps $H \dot{K}_{q_{1}, b, m}^{\alpha, p_{1}, s}\left(\mathbb{R}^{n}\right)$ into $\dot{K}_{q_{2}}^{\alpha, p_{2}}\left(\mathbb{R}^{n}\right)$ and $H K_{q_{1}, b, m}^{\alpha, p_{1}, s}\left(\mathbb{R}^{n}\right)$ into $K_{q_{2}}^{\alpha, p_{2}}\left(\mathbb{R}^{n}\right)$, respectively. 
REMARK 2. Theorem 3 is [19, Theorem 3.1] when $\tau=0, s=0, m=1$.

THEOREM 1.4. Let $p_{1}, p_{2}, q_{1}, q_{2}, \tau, \alpha, s, \gamma, T^{\tau}$ and $b$ be as in Theorem 1.3. Assume that $\left(T^{\tau}\right)^{*}\left(g_{i, \beta}\right)=C, g_{i, \beta}(x)=b^{i}(x) x^{\beta},|\beta| \leq s, i=0,1,2, \ldots, m$. Then $T_{b, m}^{\tau}\left(\mathbb{R}^{n}\right)$ maps $H \dot{K}_{q_{1}, b, m}^{\alpha, p_{1}, s}\left(\mathbb{R}^{n}\right)$ into $H \dot{K}_{q_{2}}^{\alpha, p_{2}}\left(\mathbb{R}^{n}\right)$ and $H K_{q_{1}, b, m}^{\alpha, p_{1}, s}\left(\mathbb{R}^{n}\right)$ into $H K_{q_{2}}^{\alpha, p_{2}}\left(\mathbb{R}^{n}\right)$, respectively.

Denote by $B M O$ the space of measurable functions $b$ such that

$$
\int_{B}\left|b(x)-b_{B}\right| d x<C|B|
$$

holds for all balls $B$, where $b_{B}=|B|^{-1} \int_{B} b(x) d x$ with a constant $C$ independent of $B$.

Throughout this paper, $C$ always means a constant independent of the main parameters involved, but which may be different from line to line. For any power exponent $p$ with $1 \leq p \leq \infty$, we denote the conjugate exponent $p /(p-1)$ by $p^{\prime}$.

\section{Proofs of the theorems}

First we prove two lemmas.

LEMMA 2.1. Let $1<q \leq \infty, T^{\tau}$ be the $\left(q_{1}, \tau ; s, \gamma\right)$-fractional integral type operator defined as above and $b(x) \in B M O$. If for $i=0,1, \ldots, m, a(x) b^{i}(x)$ satisfy $s$ order vanishing moments with $\operatorname{supp} a \subset B$ with the center at $x_{0}=0$ and $x \in(2 B)^{c}$, then

$$
\left|T_{b, m}^{\tau} a(x)\right| \leq C|B|^{(s+\gamma) / n+1 / q^{\prime}}\|a\|_{L^{q}\left(\mathbb{R}^{n}\right)}\left(\frac{\left|b(x)-b_{B}\right|^{m}}{|x|^{n-\tau+s+\gamma}}+\frac{\|b\|_{B M O}^{m}}{|x|^{n-\tau+s+\gamma}}\right) .
$$

Proof. Using the sth order vanishing moments of $a(x) b^{i}(x), i=0,1, \ldots, m$, we have

$$
\begin{aligned}
T_{b, m}^{\tau} a(x) & =\int_{\mathbb{R}^{n}}(b(x)-b(y))^{m} K(x, y) a(y) d y \\
& =\int_{\mathbb{R}^{n}}(b(x)-b(y))^{m}(K(x, y)-P(x, y)) a(y) d y,
\end{aligned}
$$

where $P(x, y)$ is the $(s-1)$ th order Taylor's expansion for $K(x, y)$ as a function of $y$ at $y=0$. Again, using the $s$ th order vanishing moments of $a(x) b^{i}(x), i=0,1, \ldots, m$, 
we have

$$
\begin{aligned}
T_{b, m}^{\tau} a(x) & =\int_{\mathbb{R}^{n}} \sum_{|\zeta|=s} C_{s, \zeta} \frac{\partial^{\zeta} K\left(x, y_{0}\right)}{\partial y^{\zeta}} y^{\zeta}(b(x)-b(y))^{m} a(y) d y \\
& =\int_{\mathbb{R}^{n}} \sum_{|\zeta|=s} C_{s, \zeta}\left(\frac{\partial^{\zeta} K\left(x, y_{0}\right)}{\partial y^{\zeta}}-\frac{\partial^{\zeta} K(x, 0)}{\partial y^{\zeta}}\right) y^{\zeta}(b(x)-b(y))^{m} a(y) d y,
\end{aligned}
$$

where $y_{0}$ is a point on the line segment connecting $y$ and 0 . Thus, by (ii) in Definition 1 , and since $y_{0} \in B, y \in B,|\zeta|=s,\|a\|_{L^{1}\left(\mathbb{R}^{n}\right)} \leq|B|^{1 / q^{\prime}}\|a\|_{L^{q}\left(\mathbb{R}^{n}\right)}$ and

$$
\begin{aligned}
\int_{\mathbb{R}^{n}}\left|b(y)-b_{B}\right|^{m}|a(y)| d y & \leq\|a\|_{L^{q}\left(\mathbb{R}^{n}\right)}\left(\int_{B}\left|b(x)-b_{B}\right|^{m q^{\prime}} d y\right)^{1 / q^{\prime}} \\
& \leq C\|b\|_{B M O}^{m}|B|^{1 / q^{\prime}}\|a\|_{L^{q}\left(\mathbb{R}^{n}\right)}
\end{aligned}
$$

we have

$$
\begin{aligned}
\left|T_{b, m}^{\tau} a(x)\right| \leq & C \frac{|B|^{(s+\gamma) / n}}{|x|^{n-\tau+s+\gamma}} \int_{\mathbb{R}^{n}}|b(x)-b(y)|^{m}|a(y)| d y \\
\leq & C \frac{|B|^{(s+\gamma) / n}}{|x|^{n-\tau+s+\gamma}}\left(\left|b(x)-b_{B}\right|^{m} \int_{\mathbb{R}^{n}}|a(y)| d y\right. \\
& \left.+\int_{\mathbb{R}^{n}}\left|b(y)-b_{B}\right|^{m}|a(y)| d y\right) \\
\leq & C|B|^{(s+\gamma) / n+1 / q^{\prime}}\|a\|_{L^{q}\left(\mathbb{R}^{n}\right)}\left(\frac{\left|b(x)-b_{B}\right|^{m}}{|x|^{n-\tau+s+\gamma}}+\frac{\|b\|_{B M O}^{m}}{|x|^{n-\tau+s+\gamma}}\right) .
\end{aligned}
$$

LEMMA 2.2. Let $q>0, m \in \mathbb{N} \cup\{0\}, B=B(0, r)$. Then

$$
\begin{aligned}
\Pi & =\int_{\mathbb{R}^{n} \backslash 4 B} \frac{\left|b(x)-b_{B}\right|^{m q}}{|x|^{(n-\tau+s+\gamma) q}}|x|^{\delta} d x \\
& \leq C\|b\|_{B M O}^{m q}|B|^{-((n-\tau+s+\gamma) q-\delta-n) / n} \sum_{j=2}^{\infty}(j+1)^{m q} 2^{-j((n-\tau+s+\gamma) q-\delta-n)} .
\end{aligned}
$$

PROOF.

$$
\begin{aligned}
\mathrm{II} & =\sum_{j=2}^{\infty} \int_{2^{j} r<x \mid<2^{j+1} r} \frac{\left|b(x)-b_{B}\right|^{m q}}{|x|^{(n-\tau+s+\gamma) q-\delta}} d x \\
& \leq \sum_{j=2}^{\infty} \frac{1}{\left|2^{j} r\right|^{(n-\tau+s+\gamma) q-\delta}} \int_{2^{j+1 B}}\left|b(x)-b_{B}\right|^{m q} d x \\
& =C \sum_{j=2}^{\infty} \frac{1}{\left(2^{j} r\right)^{(n-\tau+s+\gamma) q-\delta-n}} \frac{1}{\left(2^{j+1} r\right)^{n}} \int_{2^{j+1} B}\left|b(x)-b_{B}\right|^{m q} d x .
\end{aligned}
$$


When $m q \geq 1$, we have

$$
\begin{aligned}
& \frac{1}{\left(2^{j+1} r\right)^{n}} \int_{2^{j+1} B}\left|b(x)-b_{B}\right|^{m q} d x \\
& \quad \leq C \frac{1}{\left(2^{j+1} r\right)^{n}} \int_{2^{j+1} B}\left|b(x)-b_{j^{j+1} B}\right|^{m q} d x+C\left(\sum_{i=0}^{j}\left|b_{2^{i} B}-b_{2^{i+1} B}\right|\right)^{m q} \\
& \quad \leq C\|b\|_{B M O}^{m q}+C\left(\sum_{i=0}^{j}\|b\|_{B M O}\right)^{m q} \\
& \quad=C(j+1)^{m q}\|b\|_{B M O}^{m q} .
\end{aligned}
$$

When $0<m q<1$, by Hölder's inequality, we have

$$
\begin{aligned}
& \frac{1}{\left(2^{j+1} r\right)^{n}} \int_{2^{j+1} B}\left|b(x)-b_{B}\right|^{m q} d x \\
& \leq \frac{1}{\left(2^{j+1} r\right)^{n}}\left(\int_{2^{j+1} B}\left|b(x)-b_{B}\right| d x\right)^{m q}\left|2^{i+1} B\right|^{1-m q} \\
& \quad \leq\left(\frac{1}{\left(2^{j+1} r\right)^{n}} \int_{2^{j+1} B}\left|b(x)-b_{2^{j+1} B}\right| d x+\sum_{i=0}^{j}\left|b_{2^{\prime} B}-b_{2^{i+1} B}\right|\right)^{m q} \\
& \quad \leq C(j+1)^{m q}\|b\|_{B M O}^{m q} .
\end{aligned}
$$

When $m q=0$,

$$
\frac{1}{\left(2^{j+1} r\right)^{n}} \int_{\nu^{j+1} B}\left|b(x)-b_{B}\right|^{m q} d x=C .
$$

Thus, we have finished the proof of Lemma 2.2.

ProOF OF THEOREM 1.1. We need to prove that there exists a constant $C$ such that for each function $f$ in $H_{b, m}^{p_{1}, q_{1}, s}\left(\mathbb{R}^{n}\right)$,

$$
\left\|T_{b, m}^{r} f\right\|_{L^{p_{2}\left(\mathbb{R}^{n}\right)}} \leq C\|f\|_{H_{b, m}^{p_{1}, q_{1}, s}\left(\mathbb{R}^{n}\right)},
$$

where $q_{1}, q_{2}$ are as in Definition 1, that is, $1<q_{1} \leq q_{2} \leq \infty$ such that $1 / q_{1}-1 / q_{2}=$ $\tau / n\left(=1 / p_{1}-1 / p_{2}\right)$. By a standard argument, it is enough to show that there exists a constant $C$ independent of $a$ such that $\left\|T_{b, m}^{\tau} a\right\|_{L^{p_{2}\left(\mathbb{R}^{n}\right)}} \leq C$ for each $\left(p_{1}, q_{1}, s ; b\right)$-atom $a$ of order $m$.

To prove this, without loss of generality, we may suppose that supp $a \subset B$ with center at the origin. Then

$$
\begin{aligned}
\int_{\mathbb{R}^{n}}\left|T_{b, m}^{\tau} a(x)\right|^{p_{2}} d x & =\left(\int_{4 B}+\int_{\mathbb{R}^{n} \backslash 4 B}\right)\left|T_{b, m}^{\tau} a(x)\right|^{p_{2}} d x \\
& =\mathrm{I}+\mathrm{II} .
\end{aligned}
$$


By the boundedness of $T_{b, m}^{\tau}$ from $L^{q_{1}}\left(\mathbb{R}^{n}\right)$ into $L^{q_{2}}\left(\mathbb{R}^{n}\right)$ and Hölder's inequality, we have

$$
\begin{aligned}
I & \leq C\left(|B|^{1 / p_{2}-1 / q_{2}}\left(\int_{4 B}\left|T_{b, m}^{\tau} a(x)\right|^{q_{2}} d x\right)^{1 / q_{2}}\right)^{p_{2}} \\
& \leq C\left(\|b\|_{B M O}^{m}|B|^{1 / p_{1}-1 / q_{1}}\|a\|_{\left.L^{q_{1}\left(\mathbf{R}_{n}\right)}\right)}\right)^{p_{2}} \\
& \leq C\left(\|b\|_{B M O}^{m}|B|^{1 / p_{1}-1 / q_{1}}|B|^{1 / q_{1}-1 / p_{1}}\right)^{p_{2}} \\
& =C\|b\|_{B M O}^{m p_{2}} .
\end{aligned}
$$

For II, using Lemma $2.1\left(q=q_{1}\right)$, Lemma $2.2\left(\delta=0, q=p_{2}\right)$ and $\|a\|_{L^{q_{1}\left(\mathbb{R}^{n}\right)}} \leq$ $|B|^{1 / q_{1}-1 / p_{1}}$, we have

$$
\begin{aligned}
\amalg \leq & C|B|^{(s+\gamma) p_{2} / n+p_{2} / q_{1}^{\prime}}\|a\|_{L^{q_{1}\left(\mathbb{R}^{n}\right)}}^{p_{2}} \\
& \times\left(\int_{\mathbb{R}^{n} \backslash 4 B} \frac{\left|b(x)-b_{B}\right|^{m p_{2}}}{|x|^{(n-\tau+s+\gamma) p_{2}}} d x+\int_{\mathbb{R}^{n} \backslash 4 B} \frac{\|b\|_{B M O}^{m p_{2}}}{|x|^{(n-\tau+s+\gamma) p_{2}}} d x\right) \\
\leq & C|B|^{(s+\gamma) p_{2} / n+p_{2} / q_{1}^{\prime}}|B|^{\left(1 / q_{1}-1 / p_{1}\right) p_{2}} \\
& \times\|b\|_{B M O}^{m p_{2}}|B|^{-(n-\tau+s+\gamma) p_{2} / n+1} \sum_{j=2}^{\infty}(j+1)^{m p_{2}} 2^{-j\left((n-\tau+s+\gamma) p_{2}-n\right)} \\
= & C\|b\|_{B M O}^{m p_{2}},
\end{aligned}
$$

since $n /(n-\tau+s+\gamma)<p_{2}$. This concludes the proof of Theorem 1.1.

ProOF OF THEOREM 1.2. By the atom-molecule theory of Coifman and Weiss (see $[7,13,25])$ we need only to prove that there exists a constant $C$ such that for each $\left(p_{1}, q_{1}, s ; b\right)$-atom $a$ of order $m, T_{b, m}^{\tau} a$ is a $\left(p_{2}, q_{2}, s, \varepsilon\right)$-molecule, that is,

(i) $|x|^{n d} T_{b, m}^{\tau} a(x) \in L^{q_{2}}\left(\mathbb{R}^{n}\right)$;

(ii) $N_{q_{2}}\left(T_{b, m}^{\tau} a\right)=\left\|T_{b, m}^{\tau} a\right\|_{\left.L^{q_{2}\left(\mathbb{R}^{n}\right)}\right)}^{c / d}\left\|\left.x\right|^{n d} T_{b, m}^{\tau} a(x)\right\|_{L^{q_{2}\left(\mathbb{R}^{n}\right)}}^{1-c / d}=C<\infty$;

(iii) $\int_{\mathbb{R}^{n}} T_{b, m}^{\tau} a(x) x^{\beta} d x=0,|\beta| \leq s$,

where $s \geq s_{0}=\left[n\left(1 / p_{2}-1\right)\right]$,

$$
\frac{s-\tau+\gamma}{n}>\varepsilon>\max \left\{\frac{s}{n}, \frac{1}{p_{2}}-1\right\}
$$

$\left((s-\tau+\gamma) / n>1 / p_{2}-1\right.$ since $\left.p_{2}>n /(n-\tau+s+\gamma)\right), c=1-1 / p_{2}+\varepsilon$, $d=1-1 / q_{2}+\varepsilon, 0<p_{1} \leq 1,0<\gamma \leq 1, s \geq 0,1 / q_{1}-1 / q_{2}=\tau / n$.

Let supp $a \subset B$ with the center at the origin. By the boundedness of $T_{b, m}^{\tau}$ from $L^{q_{1}}\left(\mathbb{R}^{n}\right)$ into $L^{q_{2}}\left(\mathbb{R}^{n}\right)$, we have $\left\|T_{b, m}^{\tau} a\right\|_{L^{q_{2}\left(\mathbb{R}^{n}\right)}} \leq C\|b\|_{B M O}^{m}\|a\|_{L^{q_{1}\left(\mathbb{R}^{n}\right)}}$. Let

$$
\begin{aligned}
\int_{\mathbb{R}^{n}}|x|^{q_{2} n d}\left|T_{b, m}^{\mathrm{\tau}} a(x)\right|^{q_{2}} d x & =\left(\int_{4 B}+\int_{\mathbb{R}^{n} \backslash A B}\right)|x|^{q_{2} n d}\left|T_{b, m}^{\tau} a(x)\right|^{q_{2}} d x \\
& =\mathrm{I}+\mathrm{II} .
\end{aligned}
$$


For I, by the $L^{q}\left(\mathbb{R}^{n}\right)$-boundedness of $T_{b, m}^{r}$ and $\|a\|_{L^{q_{1}\left(\mathbb{R}^{n}\right)}} \leq|B|^{1 / q_{1}-1 / p_{1}}$, we have

$$
\mathrm{I} \leq C|B|^{q_{2} d}\left\|T_{b, m}^{\tau} a\right\|_{L^{q_{2}}\left(\mathbb{R}^{n}\right)}^{q_{2}} \leq C|B|^{q_{2} d}\|b\|_{B M O}^{q_{2} m}\|a\|_{L^{q_{1}\left(\mathbb{R}^{n}\right)}}^{q_{2}} .
$$

For II, by Lemma 2.1 and Lemma $2.2\left(q=q_{2}, \delta=q_{2} n d\right)$, we have

$$
\begin{aligned}
\mathrm{II} \leq & C|B|^{q_{2}(s+\gamma) / n+q_{2} / q_{1}^{\prime}}\|a\|_{L^{q_{1}\left(\mathbb{R}^{n}\right)}}^{q_{2}} \\
& \times\left(\int_{\mathbb{R}^{n} \backslash 4 B} \frac{\left|b(x)-b_{B}\right|^{q_{2} m}}{|x|^{q_{2}(n-\tau+s+\gamma)-q_{2} n d}} d x+\int_{\mathbb{R}^{n} \backslash 4 B} \frac{\|b\|_{B M O}^{q_{2} m}}{|x|^{q_{2}(n-\tau+s+\gamma)-q_{2} n d}} d x\right) \\
\leq & C|B|^{q_{2}(s+\gamma) / n+q_{2} / q_{1}^{\prime}}\|a\|_{L^{q_{1}\left(\mathbb{R}^{n}\right)}}^{q_{2}} \\
& \times\|b\|_{B M O}^{q_{2} m}|B|^{-q_{2}(n-\tau+s+\gamma) / n+q_{2} d+1} \sum_{j=2}^{\infty}(j+1)^{q_{2} m} 2^{-j\left(q_{2}((n-\tau+s+\gamma)-n d)-n\right)} \\
= & C\|b\|_{B M O}^{q_{2} m}|B|^{q_{2} d}\|a\|_{L^{q_{1}\left(\mathbb{R}^{n}\right)}}^{q_{2}},
\end{aligned}
$$

since $q_{2}(n-\tau+s+\gamma)-q_{2} n d-n=q_{2}(s+\gamma-\tau-n \varepsilon)>0$.

Thus,

$$
\begin{aligned}
N_{q_{2}}\left(T_{b, m}^{\tau} a\right) & \leq C\|b\|_{B M O}^{m c / d}\|a\|_{L^{q_{1}\left(\mathbb{R}^{n}\right)}}^{c / d}\|b\|_{B M O}^{m(1-c / d)}|B|^{d(1-c / d)}\|a\|_{L^{q_{1}}\left(\mathbb{R}^{n}\right)}^{1-c / d} \\
& =C\|b\|_{B M O}^{m}\|a\|_{L^{q_{1}\left(\mathbb{R}^{n}\right)}|B|^{1 / p_{2}-1 / q_{2}} \leq C .} \leq .
\end{aligned}
$$

We have proved (i) and (ii). By $\left(T^{\tau}\right)^{*}\left(g_{i, \beta}\right)=C,|\beta| \leq s, i=0,1,2, \ldots, m$, and the vanishing moments of $a$, (iii) is obvious.

This concludes the proof of Theorem 1.2.

Proof of TheOREM 1.3. Let $f \in H \dot{K}_{q_{1}, b, m}^{\alpha, p_{1}, s}\left(\mathbb{R}^{n}\right)$, that is, $f(x)=\sum_{j=-\infty}^{\infty} \lambda_{j} a_{j}(x)$, where each $a_{j}$ is an $m$-order dyadic central $H \dot{K}-\left(\alpha, q_{1} ; b\right)_{s}$-atom with support $B_{j}$, and $\|f\|_{H \dot{K}_{q_{1}, b, m}^{\alpha, p_{1}, s}\left(\mathbb{R}^{n}\right)} \sim\left(\sum_{j=-\infty}^{\infty}\left|\lambda_{j}\right|^{p_{1}}\right)^{1 / p_{1}}$. Then,

$$
\begin{aligned}
& \left\|T_{b, m}^{\tau} f\right\|_{\dot{K}_{q_{2}}^{\text {a,p } p_{2}}\left(\mathbb{R}^{n}\right)}^{p_{1}} \leq C\left(\sum_{k=-\infty}^{\infty} 2^{k \alpha p_{2}}\left\|\left(T_{b, m}^{\tau} f\right) \chi_{k}\right\|_{L^{q_{2}\left(\mathbb{R}^{n}\right)}}^{p_{2}}\right)^{p_{1} / p_{2}} \\
& \leq C \sum_{k=-\infty}^{\infty} 2^{k \alpha p_{1}}\left\|\left(T_{b, m}^{\tau} f\right) \chi_{k}\right\|_{L^{q_{2}}\left(\mathbb{R}^{n}\right)}^{p_{1}} \\
& \leq C \sum_{k=-\infty}^{\infty} 2^{k \alpha p_{1}}\left(\sum_{j=-\infty}^{k-2}\left|\lambda_{j}\right|\left\|\left(T_{b, m}^{\tau} a_{j}\right) \chi_{k}\right\|_{L^{q_{2}\left(\mathbb{R}^{n}\right)}}\right)^{p_{1}} \\
& +C \sum_{k=-\infty}^{\infty} 2^{k \alpha p_{1}}\left(\sum_{i=k-1}^{\infty}\left|\lambda_{j}\right|\left\|\left(T_{b, m}^{\tau} a_{j}\right) \chi_{k}\right\|_{L^{q_{2}\left(R^{n}\right)}}\right)^{p_{1}} \\
& =I_{1}+I_{2} \text {. }
\end{aligned}
$$


For $I_{2}$, by the $L^{q}\left(\mathbb{B}^{n}\right)$-boundedness of $T_{b, m}^{\tau}$ and Hölder's inequality, we have

$$
\begin{aligned}
& I_{2} \leq C \sum_{k=-\infty}^{\infty} 2^{k \alpha p_{1}}\left(\sum_{j=k-1}^{\infty}\|b\|_{B M O}^{m} \mid \lambda_{j}\left\|a_{j}\right\|_{L^{q_{1}\left(\mathbb{R}^{n}\right)}}\right)^{p_{1}} \\
& \leq C\|b\|_{B M O}^{p_{1} m} \sum_{k=-\infty}^{\infty}\left(\sum_{i=k-1}^{\infty} 2^{(k-j) \alpha}\left|\lambda_{j}\right|\right)^{p_{1}} \\
& \leq \begin{cases}C\|b\|_{B M O}^{p_{1} m} \sum_{k=-\infty}^{\infty} \sum_{j=k-1}^{\infty} 2^{(k-j) p_{1} \alpha}\left|\lambda_{j}\right|^{p_{1}} & \text { if } 0<p_{1} \leq 1 \\
C\|b\|_{B M O}^{p_{1} m} \sum_{k=-\infty}^{\infty}\left(\sum_{j=k-1}^{\infty} 2^{(k-j) p_{1} \alpha / 2}\left|\lambda_{j}\right|^{p_{1}}\right) & \\
\times\left(\sum_{j=k-1}^{\infty} 2^{(k-j) p_{1}^{\prime} \alpha / 2}\right)^{p_{1} / p_{1}^{\prime}} & \text { if } 1<p_{1}<\infty\end{cases} \\
& \leq \begin{cases}C\|b\|_{B M O}^{p_{1} m} \sum_{j=-\infty}^{\infty}\left|\lambda_{j}\right|^{p_{1}} \sum_{k=-\infty}^{j+1} 2^{(k-j) p_{1} \alpha} & \text { if } 0<p_{1} \leq 1 \\
C\|b\|_{B M O}^{p_{1} m} \sum_{j=-\infty}^{\infty}\left|\lambda_{j}\right|^{p_{1}} \sum_{k=-\infty}^{j+1} 2^{(k-j) p_{1} \alpha / 2} & \text { if } 1<p_{1}<\infty\end{cases} \\
& \leq C\|b\|_{B M O}^{p_{1} m} \sum_{j=-\infty}^{\infty}\left|\lambda_{j}\right|^{p_{1}} \text {. }
\end{aligned}
$$

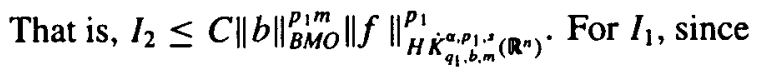

$$
\begin{aligned}
\left|b(x)-b_{B_{j}}\right|^{q_{2} m} & \leq C\left|b(x)-b_{B_{k}}\right|^{q_{2} m}+C\left(\sum_{i=j}^{k-1}\left|b_{B_{i}}-b_{B_{i+1}}\right|\right)^{q_{2} m} \\
& \leq C\left|b(x)-b_{B_{k}}\right|^{q_{2} m}+C(k-j)^{q_{2} m}\|b\|_{B M O}^{q_{2} m}
\end{aligned}
$$

and $\int_{C_{k}}\left|b(x)-b_{B_{k}}\right|^{q_{2} m} d x \leq\left|B_{k}\right|\|b\|_{B M O}^{q_{2} m} \leq C 2^{k n}(k-j)\|b\|_{B M O}^{q_{2} m}$, for $k>j$, we have

$$
\begin{aligned}
\left\|\left(T_{b, m}^{\tau} a_{j}\right) \chi_{k}\right\|_{L^{q_{2}\left(\mathbb{R}^{n}\right)}}^{q_{2}} & \\
\leq & C\left|B_{j}\right|^{(s+\gamma) q_{2} / n+q_{2} / q_{1}^{\prime}}\left\|a_{j}\right\|_{L^{q_{1}\left(\mathbb{R}^{n}\right)}}^{q_{2}} \\
& \times\left(\int_{C_{k}} \frac{\left|b(x)-b_{B_{j}}\right|^{q_{2} m}}{|x|^{q_{2}(n-\tau+s+\gamma)}} d x+\int_{C_{k}} \frac{\|b\|_{B M O}^{q_{2} m}}{|x|^{q_{2}(n-\tau+s+\gamma)}} d x\right) \\
\leq & C\left|B_{j}\right|^{(s+\gamma) q_{2} / n+q_{2} / q_{1}^{\prime}}\left\|a_{j}\right\|_{L^{q_{1}}\left(\mathbb{R}^{n}\right)}^{q_{2}} \\
& \times\left(\int_{C_{k}} \frac{\left|b(x)-b_{B_{k}}\right|^{q_{2} m}}{|x|^{q_{2}(n-\tau+s+\gamma)}} d x+\int_{C_{k}} \frac{(k-j)^{q_{2} m}\|b\|_{B M O}^{q_{2} m}}{|x|^{q_{2}(n-\tau+s+\gamma)}} d x\right)
\end{aligned}
$$




$$
\begin{aligned}
& \leq C 2^{j\left((s+\gamma) q_{2}+n q_{2}-n q_{2} / q_{1}\right)-j \alpha q_{2}} 2^{-k(n-\tau+s+\gamma) q_{2}} \times 2^{k n}(k-j)^{q_{2} m}\|b\|_{B M O}^{q_{2} m} \\
& =C 2^{(j-k)\left((s+\gamma+n) q_{2}-n q_{2} / q_{1}\right)} 2^{-j \alpha q_{2}}(k-j)^{q_{2} m}\|b\|_{B M O}^{q_{2} m} .
\end{aligned}
$$

Hence

$$
\begin{aligned}
& I_{1} \leq C\|b\|_{B M O}^{p_{1} m} \sum_{k=-\infty}^{\infty}\left(\sum_{j=-\infty}^{k-2}(k-j)^{m} 2^{(j-k)\left(n+s+\gamma-n / q_{1}-\alpha\right)}\left|\lambda_{j}\right|\right)^{p_{1}}
\end{aligned}
$$

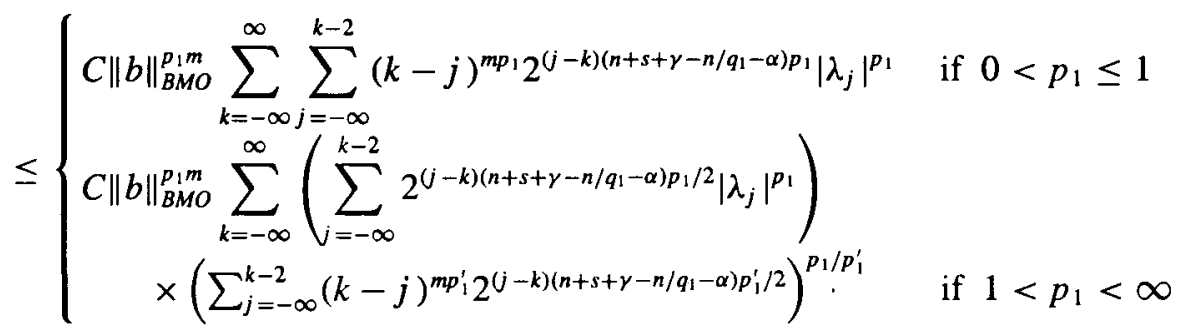

$$
\begin{aligned}
& \leq \begin{cases}C\|b\|_{B M O}^{p_{1} m} \sum_{j=-\infty}^{\infty}\left|\lambda_{j}\right|^{p_{1}} \sum_{k=j+2}^{\infty}(k-j)^{m p_{1}} 2^{(j-k)\left(n+s+\gamma-n / q_{1}-\alpha\right) p_{1}} & \text { if } 0<p_{1} \leq 1 \\
C\|b\|_{B M O}^{p_{1} m} \sum_{j=-\infty}^{\infty}\left|\lambda_{j}\right|^{p_{1}} \sum_{k=j+2}^{\infty} 2^{(j-k)\left(n+s+\gamma-n / q_{1}-\alpha\right) p_{1} / 2} & \text { if } 1<p_{1}<\infty\end{cases} \\
& \leq C\|b\|_{B M O}^{p_{1} m} \sum_{j=-\infty}^{\infty}\left|\lambda_{j}\right|^{p_{1}}
\end{aligned}
$$

for $\gamma>\alpha+n / q_{1}-n-s$. That is, $I_{1} \leq C\|b\|_{B M O}^{p_{1} m}\|f\|_{H \dot{K}_{q_{1}, b, m}^{\left.\alpha, p_{1}, \mathbb{R}^{n}\right)}}^{p_{1}}$.

Thus, we have proved the case of homogeneous spaces of Theorem 1.3 The proof of the non-homogeneous case is similar to that of homogeneous case. This concludes the proof of Theorem 1.3.

PROOF OF THEOREM 1.4. By the atom-molecule theory of $H \dot{K}_{q}^{\alpha, p}\left(\mathbb{R}^{n}\right)$ (see [18, Theorem 2.5]), we need only to prove that there exists a constant $C$ such that for each dyadic central $m$-order $H \dot{K}-\left(\alpha, q_{1} ; b\right)$-atom $a_{k}$ with support $B_{k}, T_{b, m}^{\tau} a_{k}$ is a dyadic central $\left(\alpha, q_{2}, s, \varepsilon\right)_{k}$-molecule, that is,

(i) $\left\|T_{b, m}^{\tau} a_{k}\right\|_{L^{q_{2}\left(\mathbb{R}^{n}\right)}} \leq 2^{-k \alpha}$;

(ii) $N\left(T_{b, m}^{\tau} a_{k}\right)=\left\|T_{b, m}^{\tau} a_{k}\right\|_{L^{q_{2}\left(\mathbb{R}^{n}\right)}}^{c / d}\left\||x|^{n d} T_{b, m}^{\tau} a_{k}(x)\right\|_{L^{q_{2}\left(\mathbb{R}^{n}\right)}}^{1-c / d}=C<\infty$;

(iii) $\int_{\mathbb{R}^{n}} T_{b, m}^{\tau} a_{k}(x) x^{\beta} d x=0,|\beta| \leq s$,

where $s \geq\left[\alpha+n\left(1 / q_{1}-1\right)\right],(s+\gamma-\tau) / n>\varepsilon>\max \left\{s / n, \alpha / n+1 / q_{1}-1\right\}$ (since $\left.(s+\gamma-\tau) / n>\alpha / n+1 / q_{1}-1\right), c=1-1 / q_{1}-\alpha / n+\varepsilon, d=1-1 / q_{2}+\varepsilon$.

(i) By the $L^{q}\left(\mathbb{R}^{n}\right)$-boundedness of $T_{b, m}^{\tau}$, we have

$$
\left\|T_{b, m}^{\tau} a_{k}\right\|_{L^{q_{2}\left(\mathbb{R}^{n}\right)}} \leq C\|b\|_{B M O}^{m}\left\|a_{k}\right\|_{L^{a_{1}\left(\mathbb{R}^{n}\right)}} \leq C\|b\|_{B M O}^{m} 2^{-k \alpha} .
$$


(ii)

$$
\begin{aligned}
\int_{\mathbb{R}^{n}}|x|^{q_{2} n d}\left|T_{b, m}^{\tau} a_{k}(x)\right|^{q_{2}} d x & =\left(\int_{B_{k+2}}+\int_{\mathbb{R}^{n} \backslash B_{k+2}}\right)|x|^{q_{2} n d}\left|T_{b, m}^{\tau} a_{k}(x)\right|^{q_{2}} d x \\
& =\mathrm{I}+\mathrm{II} .
\end{aligned}
$$

For I, by the $L^{q}\left(\mathbb{R}^{n}\right)$-boundedness of $T_{b, m}^{\tau}$, we have

$$
\mathrm{I} \leq C 2^{k n d q_{2}}\left\|T_{b, m}^{\mathrm{r}} a_{k}\right\|_{L^{q_{2}\left(\mathbf{R}^{n}\right)}}^{q_{2}} \leq C 2^{k n d q_{2}}\|b\|_{B M O}^{q_{2} m}\left\|a_{k}\right\|_{L^{q_{1}}\left(\mathbb{R}^{n}\right)}^{q_{2^{2}}} \leq C\|b\|_{B M O}^{q_{2} m} 2^{k q_{2}(n d-\alpha)} .
$$

For II, by Lemma 2.1 and Lemma $2.2\left(\delta=q_{2} n d\right)$, we have

$$
\begin{aligned}
& \mathrm{II} \leq C\left|B_{k}\right|^{(s+\gamma) q_{2} / n+q_{2} / q_{1}^{\prime}} \cdot\left\|a_{k}\right\|_{L^{q_{1}\left(\mathbf{R}^{n}\right)}}^{q_{2}} \\
& \times\left(\int_{\mathbb{R}^{n} \backslash B_{k+2}} \frac{\left|b(x)-b_{B_{k}}\right|^{q_{2} m}}{|x|^{q_{2}(n-\tau+s+\gamma)-q_{2} n d}} d x+\int_{\mathbb{R}^{n} \backslash B_{k+2}} \frac{\|b\|_{B M O}^{q_{2} m}}{|x|^{q_{2}(n-\tau+s+\gamma)-q_{2} n d}} d x\right) \\
& \leq C 2^{n k\left((s+\gamma) q_{2} / n+q_{2} / q_{1}^{\prime}\right)}\left\|a_{k}\right\|_{L^{q_{1}}\left(\mathbb{R}^{n}\right)}^{q_{2}} \\
& \times\|b\|_{B M O}^{q_{2} m} 2^{-n k\left((n-\tau+s+\gamma) q_{2} / n-q_{2} d-1\right)} \sum_{j=2}^{\infty}(j+1)^{q_{2} m} 2^{-j\left(q_{2}((n-\tau+s+\gamma)-n d)-n\right)} \\
& =C\|b\|_{B M O}^{q_{2} m} 2^{k q_{2} n d}\left\|a_{k}\right\|_{L^{q_{1}}\left(\mathbb{R}^{n}\right)}^{q_{2}} \sum_{j=2}^{\infty}(j+1)^{q_{2} m} 2^{-j(s+\gamma-\tau-\varepsilon n) q_{2}} \\
& =C\|b\|_{B M O}^{q_{2} m} 2^{k q_{2}(n d-\alpha)},
\end{aligned}
$$

since $(s+\gamma-\tau-\varepsilon n) q_{2}>0$. Therefore,

$$
N\left(T_{b, m}^{\tau} a_{k}\right) \leq C 2^{-k \alpha c / d} 2^{k(n d-\alpha)(1-c / d)}=C .
$$

(iii) By $\left(T^{\tau}\right)^{*}\left(g_{i, \beta}\right)=C,|\beta| \leq s, i=0,1, \ldots, m$, and the vanishing moments of $a_{k}$, the vanishing moments of $T_{b, m}^{\tau} a_{k}$ are obvious.

Thus, we have proved the case of homogeneous spaces of Theorem 1.4. The proof of the non-homogeneous case is similar to that of homogeneous case. This conclude the proof of Theorem 1.4.

\section{Fractional integrals}

Let $0 \leq \tau<n$, we define the fractional integrals of $T$ by

$$
T^{\tau} f(x)=\int_{\mathbb{R}^{n}} \frac{f(y)}{|x-y|^{n-\tau}} d x
$$

The proof of the boundedness of $T^{\tau}$ from $L^{q_{1}}\left(\mathbb{R}^{n}\right)$ into $L^{q_{2}}\left(\mathbb{R}^{n}\right)$ for $1<q_{1}<q_{2}<\infty$ such that $1 / q_{1}-1 / q_{2}=\tau / n$ can be found in [24]. Thus, the fractional integrals $T^{\tau}$ as above satisfy the condition in Definition 1 for $\gamma=1$ and any $s \geq 0$. Hence, as the special case of Theorems 1.1-1.4, we have the following corollaries. 
COROLlaRY 3.1. Let $0<p_{1} \leq 1,1 / p_{2}=1 / p_{1}-\tau / n, s>\left[n\left(1 / p_{1}-1\right)\right]$, $1<q_{1} \leq \infty$, and let $T^{\tau}$ be a fractional integral operator (as above) and $b \in B M O$. If $n /(n-\tau+s+1)<p_{2}<+\infty$ and $0 \leq \tau<n$, then $T_{b, m}^{\tau}$ maps $H_{b, m}^{p_{1}, q_{1}, s}\left(\mathbb{R}^{n}\right)$ into $L^{p_{2}}\left(\mathbb{B}^{n}\right)$.

The case $p_{1}=1$ and $m=0$ of Corollary 3.1 was proved by Stein and Weiss.

Corollary 3.2. Let $0<p_{1} \leq 1,1 / p_{2}=1 / p_{1}-\tau / n, s>\left[n\left(1 / p_{1}-1\right)\right]$, $1<q_{1} \leq \infty$, and let $T^{\tau}$ be a fractional integral operator and $b \in B M O$. Assume that $\left(T^{\tau}\right)^{*}\left(b^{i}(x) x^{\beta}\right)=C,|\beta| \leq s, i=0,1, \ldots, m$. If $n /(n-\tau+s+1)<p_{2} \leq 1$ and $0 \leq \tau<1$, then $T_{b, m}^{\tau}$ maps $H_{b, m}^{p_{1}, q_{1}, s}\left(\mathbb{R}^{n}\right)$ into $H^{p_{2}}\left(\mathbb{R}^{n}\right)$.

The case $0<p_{1}<p_{2} \leq 1$ and $m=0$ of Corollary 3.2 was proved by Taibleson and Weiss [25].

COROLlaRY 3.3. Let $0 \leq \tau<n, s \geq 0,0<p_{1} \leq p_{2}<\infty, 1<q_{1}<\infty$, $1 / q_{2}=1 / q_{1}-\tau / n, n\left(1-1 / q_{1}\right) \leq \alpha<s+1+n\left(1-1 / q_{1}\right)$, and let $T^{\tau}$ be $a$ fractional integral and $b \in B M O$. Then $T_{b, m}^{\tau}$ maps $H \dot{K}_{q_{1}, b, m}^{\alpha, p_{1}, s}\left(\mathbb{R}^{n}\right)$ into $\dot{K}_{q_{2}}^{\alpha, p_{2}}\left(\mathbb{R}^{n}\right)$ and $H K_{q_{1}, b, m}^{\alpha, p_{1}, s}\left(\mathbb{R}^{n}\right)$ into $K_{q_{2}}^{\alpha, p_{2}}\left(\mathbb{R}^{n}\right)$, respectively.

COROLlaRY 3.4. Let $p_{1}, p_{2}, q_{1}, q_{2}, \tau, \alpha, s, T^{\tau}$ and b be as in Corollary 3.3. Assume that $\left(T^{\tau}\right)^{*}\left(b^{i}(x) x^{\beta}\right)=C,|\beta| \leq s, i=0,1, \ldots$, m. Then $T_{b, m}^{\tau}$ maps $H \dot{K}_{q_{1}, b, m}^{\alpha, p_{1}, s}\left(\mathbb{R}^{n}\right)$ into $H \dot{K}_{q_{2}}^{\alpha, p_{2}}\left(\mathbb{R}^{n}\right)$ and $H K_{q_{1}, b, m}^{\alpha, p_{1}, s}\left(\mathbb{R}^{n}\right)$ into $H K_{q_{2}}^{\alpha, p_{2}}\left(\mathbb{R}^{n}\right)$, respectively.

The case $m=0$ of Corollary 3.3 and Corollary 3.4 can be found in $[14,18]$.

The case $m=1$ of Corollary 3.3 can be found in [19].

\section{Acknowledgement}

The authors wish to express their deep thanks to the referee and Professor A. H. Dooley for many valuable comments.

\section{References}

[1] J. Alvarez, 'Continuity properties for linear commutators of Calderón-Zygmund operators', Collect. Math. 48 (1998), 17-31.

[2] J. Alvarez, R. J. Bagby, D. S. Kurtz and C. Perez, 'Weighted estimates for commutators of linear operators', Studia Math. 104 (1993), 195-209.

[3] S. Bloom, 'A commutator theorem and weighted BMO', Trans. Amer. Math. Soc. 292 (1985), 103-122.

[4] S. Chanillo, 'A note on commutators', Indiana Univ. Math. J. 31 (1982), 7-16. 
[5] Y. Chen and K. Lau, 'On some new classes of Hardy spaces', J. Funct. Anal. 84 (1989), 225-278.

[6] R. R. Coifman, R. Rochberg and G. Weiss, 'Factorization theorems for Hardy spaces in several variables', Ann. of Math. (2) 103 (1976), 611-635.

[7] R. R. Coifman and G. Weiss, 'Extensions of Hardy spaces and their use in analysis', Bull. Amer. Math. Soc. 83 (1977), 569-645.

[8] Y. Ding and S. Lu, 'Higher order commutators for a class of rough operators', Ark. Mat. 37 (1999), $33-44$.

[9] J. Garcia-Cuerva, 'Hardy spaces and Beurling algebras', J. London Math. Soc. 39 (1989), 499-513.

[10] J. Garcia-Cuerva, E. Harboure, C. Segovia and J. Torrea, 'Weighted norm inequalities for commutators of strongly singular integrals', Indiana Univ. Math. J. 40 (1991), 1397-1420.

[11] L. Grafakos, X. Li and D. Yang, 'Bilinear operators on Herz-type spaces', Trans. Amer. Math. Soc. 350 (1998), 1249-1275.

[12] G. Hu, S. Lu and D. Yang, 'Boundedness of rough singular integral operators on homogeous Herz spaces', J. Austral. Math. Soc. (Ser. A) 66 (1999), 201-233.

[13] R. H. Latter, 'A decomposition of $H^{p}\left(\mathbb{R}^{n}\right)$ in terms of atoms', Studia Math. 62 (1977), 92-101.

[14] X. Li and D. Yang, 'Boundedness of some sublinear operators on Herz spaces', Illinois J. Math. 40 (1996), 484-50l.

[15] S. Long and J. Wang, 'The higher order commutator theorem on Hardy spaces', preprint, 1999.

[16] S. Lu and D. Yang, 'The local versions of $H^{p}\left(\mathbb{R}^{n}\right)$ spaces at the origin', Studia Math. 116 (1995), 103-131.

[17] — 'Weighted Hardy spaces associated with Herz spaces and its applications', Sci. China (Ser. A) 3 (1995), 235-245 in Chinese.

[18] — 'Hardy-Littlewood-Sobolev theorem of fractional integration on Herz-type spaces and its applications', Canad. J. Math. 48 (1996), 363-380.

[19] — 'The continuity of commutators on Herz-type spaces', Michigan Math. J. 44 (1997), 255-281.

[20] M. Paluszynski, Characterization of Lipschitz spaces via commutator of Coifman, Rochberg and Weiss: a multiplier theorem for the semigruop of contractions (Ph.D. Thesis, Washington Univiversity, St. Louis, 1992).

[21] C. Perez, 'Endpoint estimates for commutators of singular integral operators', J. Funct. Anal. 128 (1995), 163-185.

[22] C. Segovia and J. L. Torrea, 'Vector-valued commutators and applications', Indiana Univ. Math. J. 38 (1989), 959-971.

[23] —. 'Higher order commutators for vector-valued Calderón-Zygmund operators', Trans. Amer. Math. Soc. 336 (1993), 537-556.

[24] E. M. Stein, Singular integrals and differentiability propertites of functions (Princeton Univ. Press, Princeton, NJ, 1970).

[25] M. Taibleson and G. Weiss, 'The molecular characterization of certain Hardy spaces', Asterisque 77 (1980), 67-151.

\section{Mathematics Department}

Xiangtan University

Xiangtan, 411105

P. R. China

e-mail: sclong@xtu.edu.cn, jwang@hnedu.com 